\title{
Driving behaviour in a real and a simulated road tunnel - a validation study
}

Reprint from Accident \& Analysis Prevention, Vol. 30, No. 4, pp. 497-503, 1998

Jan Törnros

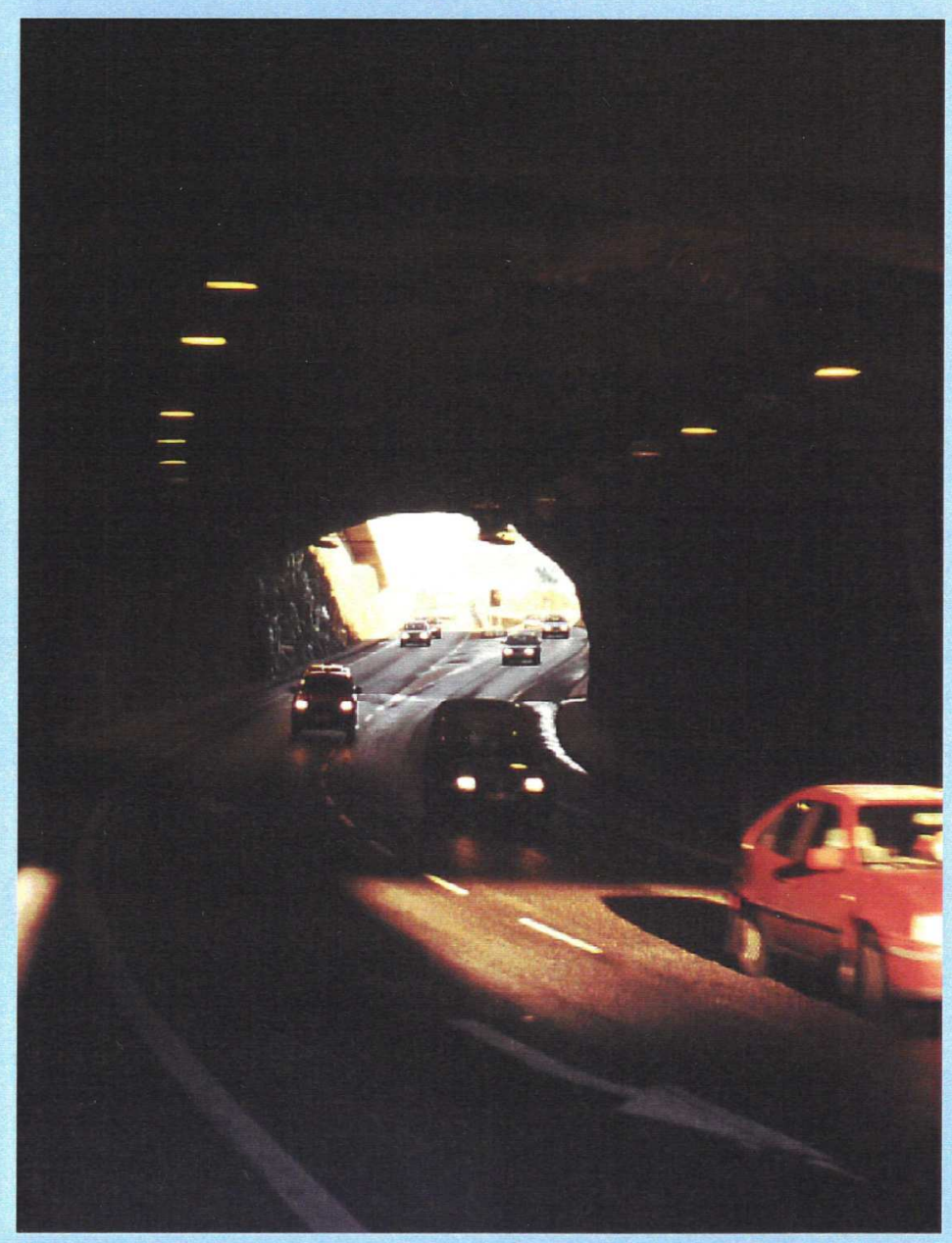

ab 


\section{VTI särtryck $301 \cdot 1998$ \\ Driving behaviour in a real and a simulated road tunnel - a validation study}

Reprint from Accident \& Analysis Prevention, Vol. 30, No. 4, pp. 497-503, 1998

Jan Törnros 


\title{
DRIVING BEHAVIOUR IN A REAL AND A SIMULATED ROAD TUNNEL-A VALIDATION STUDY
}

\author{
JAN TÖRNROS
}

\begin{abstract}
Swedish Road and Transport Research Institute (VTI), S-581 95 Linköping, Sweden
\end{abstract}
(Received 10 February 1997; in revised form 16 September 1997)

\begin{abstract}
The aim was to validate driving behaviour in a simulated road tunnel. Speed and lateral position of 20 subjects were measured in a real tunnel and in the same tunnel implemented in the VTI driving simulator. In both situations a left-hand steered and manually geared passenger car was used. Driving speed was higher in the simulated tunnel than in the real tunnel. Elimination of speed information from the speedometer caused a similar small speed increase in both situations. Also, the difference in speed between driving lanes was similar in both cases. The effects on speed variation were similar to that for speed level. Regarding lateral position, subjects positioned the car somewhat further away from the nearest tunnel wall in the real tunnel than in the simulated tunnel. In both situations the distance to the nearest wall was greater when it was located to the left of the driver than on the opposite side. Lateral position deviation was about the same when the road was straight, but in a curved section it was somewhat greater for the simulated tunnel. It is concluded that behavioural validity in absolute terms was not quite satisfactory, especially regarding choice of speed, whereas relative validity was good for both speed and lateral position. (C) 1998 Elsevier Science Ltd. All rights reserved
\end{abstract}

Keywords-Tunnel, Driving, Simulation, Validation

\section{INTRODUCTION}

The VTI driving simulator is at present being used as a planning tool in road-tunnel design (Nordmark et al., 1995). For this to be a meaningful endeavour it is essential that the correspondence between a real and a simulated tunnel is sufficiently good. It is of special importance that road-user behaviour is sufficiently similar in both situations; i.e., it is essential that the driving simulator is sufficiently valid with respect to driving behaviour.

This predictive validity can be described from two aspects, absolute and relative validity. The former refers to the numerical correspondence between behaviour data in the driving simulator and in the real situation, whereas relative validity refers to the correspondence between effects of different variations in the driving situation (Harms, 1994).

For a driving simulator to be useful as a research tool it is necessary that the relative validity is satisfactory, i.e. the same, or at least similar, effects are obtained in both situations. Absolute validity is not a necessary requirement, since research questions almost uniquely deal with matters relating to effects of various independent variables.

In order to obtain a multifaceted view of the predictive validity of a driving simulator with respect to driving behaviour in road tunnels, it would be desirable to study this behaviour in different driving situations in different kinds of tunnels. For various reasons this cannot be done, which, of course, restricts the generalisation possibilities. Analogously it is not possible to generalise to other behavioural measures beyond those actually selected for study.

In order to keep the experimental situation as well controlled as possible, it was decided to study driving behaviour in a tunnel completely free of other traffic. A newly built road tunnel, the Ekeberg tunnel in Oslo, Norway, which was just about to be opened for traffic, was selected for the study. Effect measures were driving speed and lateral position.

One aim was to study the absolute validity but, more importantly, the relative validity, in this situation.

\section{METHOD}

\section{Apparatus}

The VTI driving simulator (Fig. 1) and an instrumented car were used. The driving simulator is an advanced construction consisting of six subsystems: a computer system with its simulation model; a 


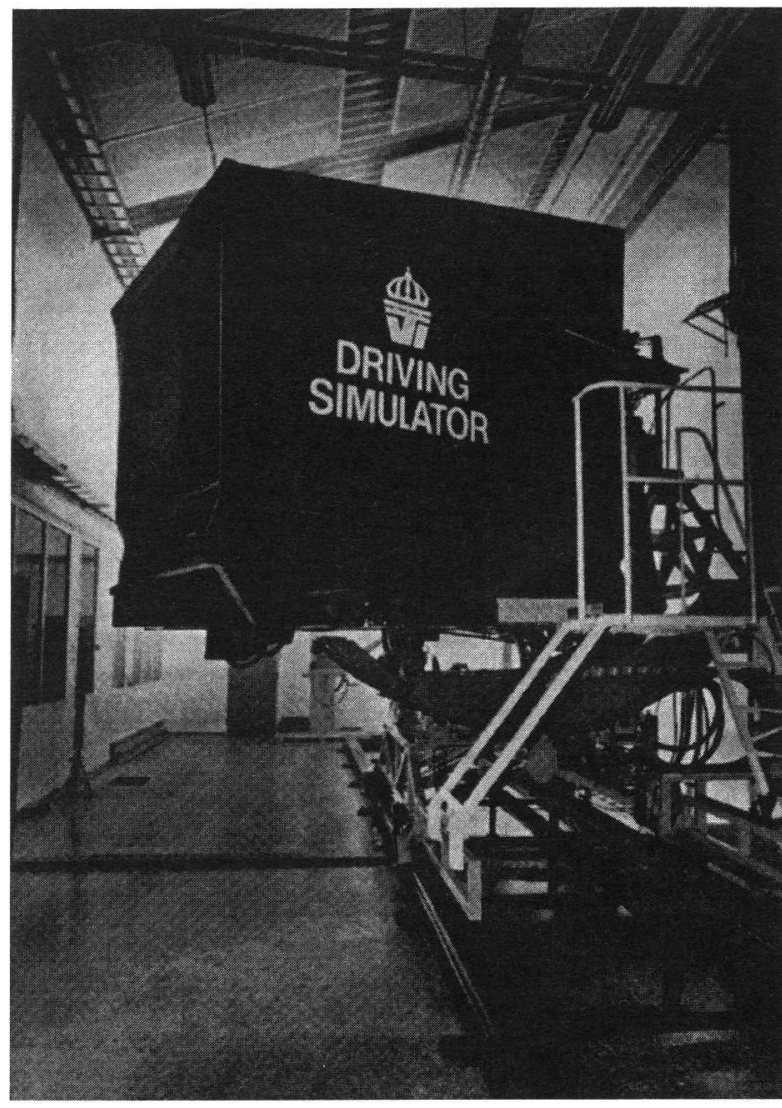

Fig. 1. VTI driving simulator (photograph: Bengt Arne Ignell).

moving base system simulating acceleration in different directions; a visual system with its three video projectors presenting the road, other traffic and surroundings onto a $120^{\circ}$ wide screen; a vibration system simulating the vibrations caused by the roadvehicle contact; a sound system; and, finally, a temperature system (Nilsson, 1989, 1993; Nordmark, 1994). These subsystems are controlled to interact in a way that will give the driver the impression that he is actually driving a real car on a real road.

The whole capacity of the moving base system was not used in the study. The reason was that a few subjects experienced symptoms of motion sickness when driving the simulator, supposedly caused by too many sharp accelerations and decelerations in too short a time. For that reason the simulated forces in the driving direction were abandoned entirely. After that, no one suffered from motion sickness. The five individuals who had performed their driving sessions with the whole moving base system in action repeated their simulated driving session after this modification. Only data from this later session were used in the analyses.

The cabin of the driving simulator was a lefthand steered SAAB 9000.

The instrumented car was a left-hand steered SAAB 9000 CDE (1995) - a car with a very powerful engine, identical to the engine of the vehicle model used in the simulation. It was, like the simulator, manually geared and equipped with apparatus for measuring speed and lateral position. The latter was measured as the distance between a point straight under a video camera mounted on the roof of the car and the side lines of the road. Data were transformed to an average (arithmetic mean) value for every $5 \mathrm{~m}$ and were stored for later analysis.

In the driving simulator, speed and lateral position were measured with a sampling frequency of $10 \mathrm{~Hz}$.

\section{Tunnel section}

The testing was performed in a section of the Ekeberg tunnel, which was newly built and had not yet opened for traffic (Fig. 2). This tunnel was also implemented in the VTI driving simulator, based upon available design drawings and video recordings.

Figures 3 and 4 show a section of the tunnel, in the real and the simulated cases, respectively.

The tunnel walls were made of concrete with visible repeated joinings between concrete elements. Due to some visual distortion, the joinings were simulated on the middle screen only, subtending $\pm 20^{\circ}$ of the whole screen. Further out peripherally only a uniform dark grey surface was presented. All installations mounted to the walls were, however, simulated all the way out into the peripheries $\left( \pm 60^{\circ}\right)$.

The ceiling of the tunnel was dark, with lamps mounted in rows in the driving direction. The signed speed limit was $70 \mathrm{~km} /$ hour.

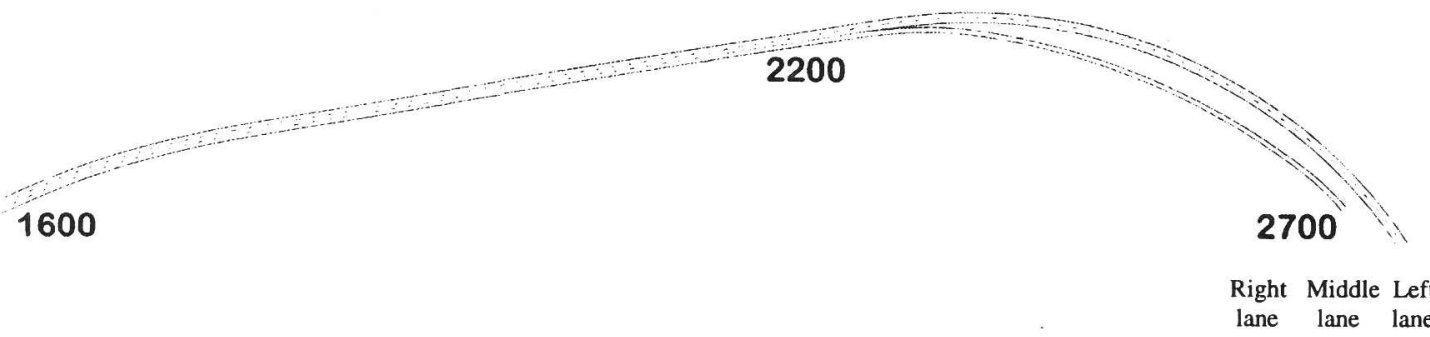

Fig. 2. Tunnel with approximate distances (m), seen from above 


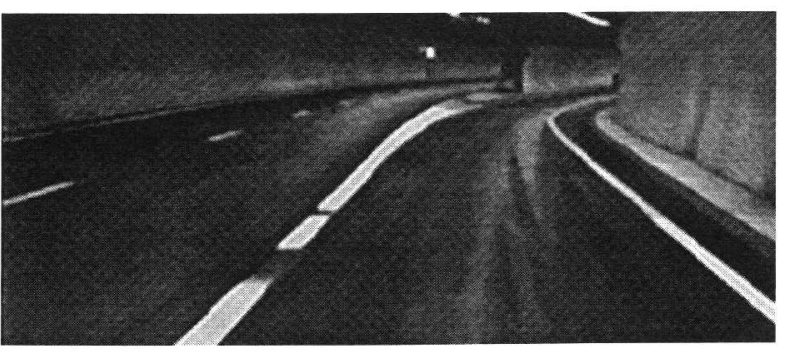

Fig. 3. The real tunnel.

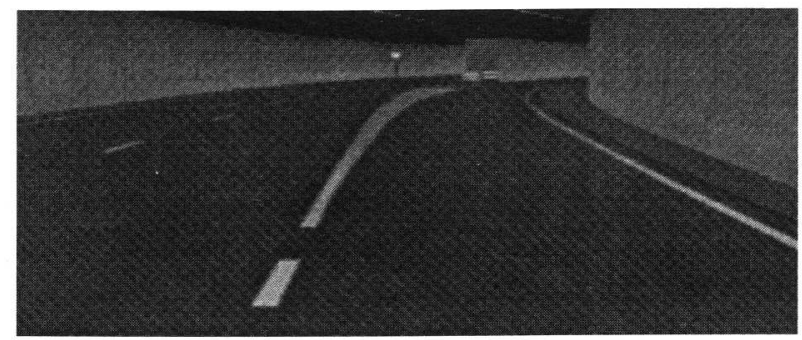

Fig. 4. The simulated tunnel.

The width of the driving lanes varied:

(1) The left lane was $3.25-\mathrm{m}$ wide on the straight section before the long curve (radius $375 \mathrm{~m}$ ), where the lane was widened to $3.65 \mathrm{~m}$.

The middle lane was $3.50-\mathrm{m}$ wide on the straight section before the long curve, where the lane was widened to $3.65 \mathrm{~m}$.

The right (exiting) lane had the same width as the left lane on the straight section before the long curve, where the lane was widened to $3.75 \mathrm{~m}$.

The measurement distance was $1,150 \mathrm{~m}$ for the left and the middle lanes, and $980 \mathrm{~m}$ for the right lane.

The tunnel sloped somewhat in the direction of travel, from $4.5 \%$ upwards to $2.6 \%$ downwards. The same slope conditions applied for the simulated tunnel. The slope conditions affected the speed of the engine in a realistic manner. The real tunnel was also slanted sideways, maximally $7 \%$, whereas the simulated tunnel was not slanted at all in that direction.

Driving behaviour was measured in both directions.

\section{Subjects}

Twenty subjects (9 men, 11 women), all with valid driving licences, participated as paid subjects in the study. Ages varied between 23 years and 52 years (average 38.5 years). Their self-reported annual driving distance varied between 3,000 and $60,000 \mathrm{~km}$, with $18,250 \mathrm{~km}$ on average.

\section{Experimental design}

A repeated measurements design was used. All 20 subjects thus participated in all experimental conditions.
Each subject drove through the tunnel 12 times, six times in each direction. Two of these rides were made in each of the three driving lanes, in one case with speedometer information and the other without that information available.

In order to calculate the test-retest reliability, each subject made an extra ride through the tunnel in each direction in one of the driving lanes. In one case speed information was provided from the speedometer, but in the other case it was not.

The same design was used in the real and in the simulated cases.

Order effects were controlled: the order of driving lanes was balanced across subjects. Half of the subjects had access to speed information from the speedometer in the first six trials, whereas this was not the case in the remaining six trials. For the other half of the subjects the order of presentation was reversed.

Shortage of time made it necessary that all the driving sessions in the real tunnel were done before using the simulated tunnel. Consequently, the control of position effects are not satisfactory regarding comparisons between the simulated and the real tunnel, bearing on matters of absolute validity. This lack of control is not regarded as a real problem for comparisons of effects of factors affecting driving behaviour in the real and simulated case, i.e. matters of relative validity.

Apart from comparisons relating to absolute validity, in order to gain insight into matters of relative validity effects of some factors were studied. For speed data the following two factors were studied: access to speed information from the speedometer and driving lanes. A reasonable hypothesis for the first variable is that removal of such information will increase speed. For the second factor, driving lanes, speed should be lowest in the right lane, since this lane departs from the rest of the tunnel and runs in a comparatively narrow tunnel with somewhat smaller curve radius at the entrance to this more narrow section.

For lateral position, the independent variables were: location of the tunnel wall and curvature. For the first of these two factors, the hypothesis is that, assuming that drivers position the car at a safe distance away from the tunnel wall, subjects will stay a greater distance from the wall when it is on their left side than when it is on their right (assuming that the car is left steered). The second factor, curve radius, ought to affect lateral position considering the tendency to follow the inner side of the curve. An interaction between curve radius and the location of the tunnel wall can also be expected.

\section{Procedure}

All subjects made all their test drives in the real tunnel before those in the simulated one. The time 
interval between the two occasions was about 3 months.

Each subject started the session by driving the car for about 20 minutes outside the tunnel. Three practise drives were made through the tunnel before measurements began.

Subjects were instructed to drive as they normally do in a tunnel with a speed limit $70 \mathrm{~km} /$ hour.

Instructions and procedures were the same in the simulated and the real cases.

\section{Data analysis}

All behavioural data were analysed by analysis of variance (repeated measurement). Main effects of the different factors and interactions were analysed. A $95 \%$ significance level was adopted in all cases $(\alpha=0.05)$.

For every statistically significant $F$ value, $\omega^{2}$ (omega squared) was calculated as a measure of explained variance (Keppel, 1982). Statistically significant interactions were followed up by analysing simple effects. In order to control the $\alpha$ level, significance levels were increased according to recommendations by Kirk (1968).

\section{RESULTS}

\section{Speed}

Effects of the following factors were analysed through analysis of variance:

simulator (simulated tunnel or real tunnel);

lane (left, middle, or right driving lane);

speedometer (with or without speedometer information).

Figure 5 shows that the speed was higher in the simulated tunnel than in the real one; the difference was $8.6 \mathrm{~km} /$ hour. This difference is statistically significant $(p<0.001) . \omega^{2}=0.36$, which means that more than one-third of the total variation of the effect measure is explained by this factor. The result can be seen as a sign of lacking absolute validity.

The difference between driving lanes is also statis-

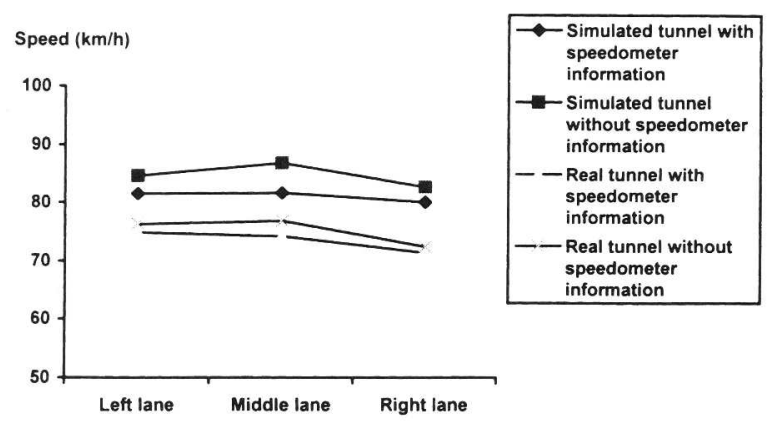

Fig. 5. Average speed $(\mathrm{km} / \mathrm{h})$ in the real and simulated tunnels. tically significant $(p<0.01)$. Four percent of the variation is explained by this factor.

Pairwise comparisons (Tukey) show that the speed was lower in the right driving lane than in the other two lanes (left lane-middle lane: $N S$; left laneright lane: $p<0.01$; middle lane-right lane: $p<0.01)$. The difference between the right lane and the other two lanes was $2.9 \mathrm{~km} /$ hour on the average.

There is no interaction between the simulator factor and the lane factor, however, which means that the effect of the lane factor applies to both the simulated and the real tunnel. This can be seen as a sign of good relative validity.

Finally, an effect of speedometer information is evident $(p<0.01)$. Three percent of the variation is explained by this factor. The speed was $2.7 \mathrm{~km} /$ hour lower when speed information was available than when this was not the case.

No interaction appears between the simulator factor and the speedometer factor, which means that the effect of the speed information applies to both situations. This can, once again, be seen as a sign of good relative validity.

No other effects were found for this measure.

\section{Lateral position}

The aim was to determine the effect of the location of the tunnel walls. The lateral position measure consisted of the distance between the middle of the car and the middle of the side line closest to the nearest tunnel wall.

The width of the driving lanes varied between different parts of the tunnel (see above). Since this fact could be expected to affect lateral position, comparisons were made only for stretches with the same lane widths. Lateral position was thus analysed separately for straight sections and for curved sections. For the straight section, comparisons were made only between the left and the right lanes, excluding the middle lane. For the long curved section, comparisons were made only between the left and the middle lanes, thus excluding the right (exiting) lane.

Straight section. Effects of two factors were analysed (analysis of variance):

simulator (simulated tunnel or real tunnel); tunnel wall (nearest wall located to the left or to the right).

Figure 6 shows that the subjects positioned themselves further from the side line in the real tunnel than in the simulated one. The average difference was $13 \mathrm{~cm}$, a statistically significant result $(p<0.001)$. Seven percent of the variation of the effect measure is explained by this factor.

The difference between the tunnel wall located 


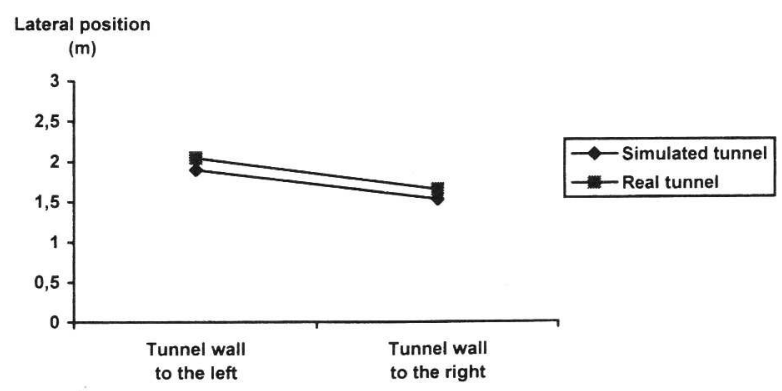

Fig. 6. Average lateral position (m) in the real and simulated tunnels on straight section.

to the left and to the right is also statistically significant $(p<0.001)$, which means that the subjects positioned the car further from the side line when the nearest tunnel wall was on their left side. The average difference was $39 \mathrm{~cm}$. The effect is large, explaining $52 \%$ of the variation of the effect measure.

On the other hand, there is no interaction between the simulator factor and the tunnel wall factor. The difference between the tunnel wall locations $(39 \mathrm{~cm})$ thus applies to both situations, which can be seen as a sign of good relative validity.

Curved section. Effects of three factors were analysed (analysis of variance):

simulator (real tunnel or simulated tunnel); tunnel wall (nearest wall located to the left or to the right);

curve (left curve or right curve).

Figure 7 shows the lateral position for the real and simulated tunnels. The results are shown separately for left and right curve and for location of the nearest tunnel wall.

The difference between the real and simulated tunnels is statistically significant $(p<0.001)$. Ten percent of the variation of the effect measure is explained by this factor. On average, the subjects positioned themselves $13 \mathrm{~cm}$ further away from the side line in the real tunnel.

The difference between the location of the tunnel wall is also statistically significant $(p<0.001)$.

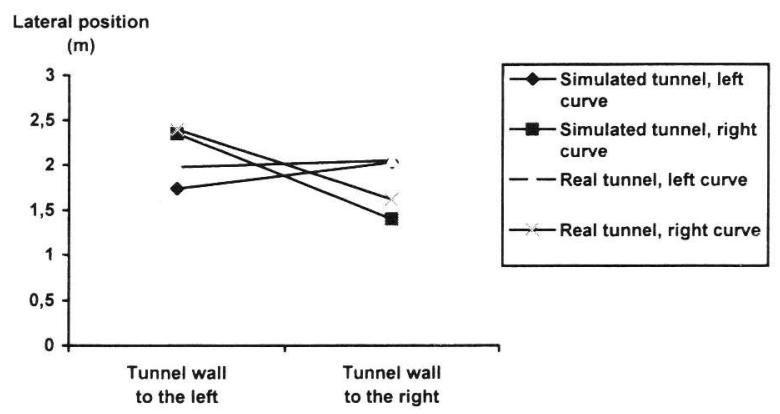

Fig. 7. Average lateral position $(\mathrm{m})$ in the real and simulated tunnels on curved section.
Nineteen percent of the variation is explained by this factor. The subjects thus positioned the car further away from the side line when the nearest tunnel wall was to the left than when it was to the right. The average difference was $34 \mathrm{~cm}$.

There is no interaction between the simulator factor and the tunnel wall factor. The difference between the locations of the tunnel wall $(34 \mathrm{~cm})$ thus applies to both the real and simulated cases, again showing signs of good relative validity.

There is no main effect of the curve factor, and no interaction between the curve factor and the simulator factor. The interaction between tunnel wall and curve is, however, statistically significant $(p<0.001)$. The effect is large, accounting for $47 \%$ of the variance of the effect measure. The triple interaction simulator $\times$ tunnel wall $\times$ curve is also statistically significant $(p<0.01)$. Although this interaction is weak, accounting for only $1 \%$ of the variance, it indicates that the tunnel wall $\times$ curve interaction is somewhat different in the real and the simulated situations.

Analyses of simple interaction effects show that the tunnel wall $\times$ curve interaction is statistically significant in both cases $(p<0.01)$. Analysis of simple main effects for the simulated tunnel shows that when driving in the left curve, subjects positioned the car further away from the side line when the nearest tunnel wall was to the right than to the left $(p<0.01)$. In the right curve the result was the opposite $(p<0.01)$.

It was also found that when the nearest wall was to the left, the subjects positioned the car further from the side line when driving in the right curve compared to driving in the left curve $(p<0.01)$. When the nearest tunnel wall was to the right, the result was the opposite $(p<0.01)$.

Analysis of simple main effects for the real tunnel gave the same result, with one exception; when driving in the left curve the difference between the nearest wall to the left and to the right was not statistically significant. The same tendency, however, appeared as for the simulated tunnel.

The triple interaction found is so weak that it can hardly affect the conclusion that the results, once again, can be seen as a sign of good relative validity.

\section{Reliability}

Test-retest reliability was calculated for the two behaviour measures. Reliability was good in the real tunnel ( 0.96 for speed and 0.97 for lateral position), but lower in the simulated one $(0.87$ for speed and 0.78 for lateral position). 


\section{DISCUSSION}

A relatively large number of significance tests were made, each one at $95 \%$ significance level. The larger the number of such tests, the greater is the risk for false significance. Therefore individual statistically significant results should be interpreted with some care.

One of the results was that speed was higher in the simulated tunnel.

Two validation studies have been performed previously on the VTI driving simulator. Driving behaviour was measured in the same way as in the present study. Rural road driving was studied in both cases. The road was the same in both cases (an $8-\mathrm{km}$ stretch of paved country road). The first of these studies (Harms, 1994) used a Volvo 240 Sedan (1984), whereas the driving simulator had different characteristics; the cabin was a SAAB 9000, while the vehicle model was adjusted to a different car make. There was other traffic in the real case, but not so in the simulated case. Speed level was found to be comparable in both situations. However, subjects positioned themselves further away from the middle of the road in the real case than in the simulated one. The reason behind this result was unclear, but the author put forward the hypothesis that it could be due to the fact that there was other traffic only on the real road. Another possible explanation was given in terms of cues used for choosing lateral position.

In the second validation study (Alm, 1995), all other traffic appearing on the real road was simulated. This time a SAAB 9000 was used, which corresponded to the cabin used in the driving simulator. The vehicle model was the same as in the first study. Lateral position was not markedly different this time, which, the author suggested, could be a result of the presence of other traffic in both situations. Speed level was about the same in the simulated and the real case. Speed variation was, however, somewhat higher in the simulated situation.

The reason why speed differed so markedly in the present study but not in the other two studies is unclear and not easily explained, since several factors differed in the three studies (traffic environment, cars, vehicle models, subjects). Some possible explanatory factors could be proposed, though. One is the difference in peripheral speed information; in the first two studies, simulating rural driving, more such information was presented than in the present case. Peripheral flow of information is believed to be important for the perception of speed (Shinar, 1978), and might at least partly explain the lower speed in the real tunnel. Other factors may have contributed, especially the power of the engine (which the subjects were generally unaccustomed to) in combination with the absence of simulation of longitudinal forces. The comparatively short stretch of the test road and subjects' relative lack of experience in tunnel driving may have played a part. The result might also, to some extent, be a result of presentation order; it is possible that some subjects drove faster in the driving simulator when they realised that the test session was simply a (perhaps not very entertaining) repetition of what had been done in the real tunnel, and speeding was a way to end the session quickly.

It is interesting to note, however, that the speedometer information as well as driving lanes had similar effects in both situations. Speedometer information was evidently useful in both cases. The effect of driving lanes is probably explained by the fact that the right (exiting) lane departed from the rest of the tunnel and ran in a much more narrow tunnel section towards the end, causing a speed reduction.

The results regarding lateral position can probably be explained as an effect of the tunnel walls, even if no such effect can be isolated in a strict sense.

Subjects thus positioned the car much further away from the wall when it was to the left than on the opposite side. This is very obvious for the straight tunnel section. In the curved section the lateral position is evidently affected by the tendency to 'cut' the curve. In the right curve this effect is strengthened, and in the left curve the result is the opposite. Taken together, however, the result for the curved section is basically the same as that for the straight section. All these effects apply to both the real and the simulated tunnel.

The distance to the side line was, in general, somewhat larger in the real tunnel. A reasonable interpretation is that the tunnel wall had greater effects in the real tunnel than in the simulated tunnel.

Taken together, the speed was higher in the simulated tunnel than in the real tunnel. In this situation subjects drove closer to the tunnel wall than in the real tunnel. Absolute validity thus seems to be lacking somewhat, especially regarding speed choice.

However, it is clear that the effects on speed and lateral position are mostly very similar in the two situations, which means that relative validity seems satisfactory with respect to the measures used.

These findings indicate that research questions dealing with matters related to absolute validity regarding speed or lateral position can be expected to give less valid results in this driving simulator with this particular tunnel configuration with this particular car. However, the results indicate that effects of various independent factors found in the driving simulator may well be generalisable to the real situa- 
tion, which suggests that the driving simulator is a useful research tool in this area. It should be pointed out, however, that it is not possible in a strict sense to generalise from the present case to other situations or effect measures.

Because of control problems (the effects of order of presentation between real and simulated tunnel uncontrolled), performed calculations of absolute validity are uncertain. It is possible that the differences between the real and the simulated tunnel might be smaller in reality than was found in the present study, at least regarding the speed measure.

In conclusion, in spite of some differences between the results for the real and the simulated tunnel, indicating that absolute validity is lacking somewhat, the findings with respect to relative validity seem promising for the driving simulator as a useful and valuable research tool in this area of research.

Acknowledgements-This work was supported by the Swedish National Road Administration, Stockholm.

\section{REFERENCES}

Alm, H. (1995) Driving simulators as research tools - a validation study based on the VTI Driving Simulator. In: DRIVE II V2065: GEM Validation Studies-Appen- dix, Reference. GEM/TR/TRC/MK950327, Work package 500, Deliverable No. 10, Rover, British Aerospace, Philips Research Laboratories, TNO Institute for Perception, Kara (COSILY), ACit GmbH, TRC University of Groningen, University of Leeds, VTI, 1995.

Harms, L. (1994) Driving performance on a real road and in a driving simulator: results of a validation study. In: Gale, A. G. (ed.), Vision in Vehicles, Vol. V. Elsevier, North Holland.

Keppel, G. (1982) Design and Analysis-A Researcher's Handbook. Prentice-Hall, Englewood Cliffs, NJ.

Kirk, R. E. (1968) Experimental Design: Procedures for the Behavioral Sciences. Brook/Cole, Belmont, CA.

Nilsson, L. (1989) The VTI driving simulator. Description of a research tool. Drive project V1017 (Bertie), 1989. VTI särtryck 150.

Nilsson, L. (1993) Behavioural research in an advanced driving simulator - experiences of the VTI System. In Proceedings of the Human Factors and Ergonomics Society, 37th Annual Meeting, Seattle, WA. VTI särtryck 197.

Nordmark, S. (1994) Driving simulators, trends and experiences. RTS'94 Driving Simulation Conference, Paris. VTI särtryck 204.

Nordmark, S., Jansson, H., Lidström, M. and Palmkvis, G. (1995) Driving simulators in tunnel design-experiences of a new tool. In: Vardy, A. E. (ed.), Safety in Road and Rail Tunnels. Proceedings of the Second International Conference, Granada.

Shinar, D. (1978) Psychology on the Road. The Human Factor in Traffic Safety. John Wiley, New York. 
VI FORSKAR FÖR ETT LIV I RÖRELSE

Statens väg- och transportforskningsinstitut (VTI) har kompetens och laboratorier för kvalificerade forskningsuppdrag inom transporter och samhällsekonomi, trafiksäkerhet, fordon, miljö samt för byggande, drift och underhăll av vägar och järnvägar.

The Swedish National Road and Transport Research Institute (VTI) has laboratories and know-how for advanced research commissions in transport and welfare economics, road safety, vehicles and the environment. It also has research capabilities for the construction, operation and maintenance of roads and railways.

\section{Adress}

Postal address

SE-581 95 Linköping, Sweden
Telefon

Telephone

Nat 013-20 4000

Int +4613204000
Fax

E-post

E-mail

Nat 013-14 1436

Int +4613141436 